\title{
Final Technical Report for:
}

\section{"Scalable Dynamic Mesh Algorithms and Software"}

\section{September 1, 1998 - November 30, 2001}

PIs: Mark Jones - The Bradley Department of Electrical Engineering, Virginia Tech

Paul Plassmann - Department of Computer Science and Engineering, Penn State

Development of compression algorithms for time-dependent mesh data: A common problem for large-scale, time-dependent scientific simulations is the storage of the vast amount simulation data generated.

We have developed algorithms and software for the time (and space) compression of mesh-based data. These new algorithms exhibit approximately an order of magnitude of compression in the data set sizes. These algorithms allow mesh (or other) data from a parallel computer to be compressed for viewing on a visualization workstation via fast uncompression methods or for archiving on disk storage. A key feature of these algorithms is that they expend more effort on the compression end (using the power of the parallel computer), while requiring a small amount of computation for the uncompression phase.

However, even given the lighter computational requirements of uncompression, there is still a significant computational load on the visualization workstation as it tries to handle data from hundreds of processors. Accelerator boards; in the form of configurable computers, have proven very successful in many applications. The boards typically sit on the PCI bus and provide significant computational capacity for a host computer. To take advantage of these new architectures, we mapped the uncompression algorithms to two configurable computer architectures (the AMS WildForce board and the SLAAC-1V board). These boards gain their computational advantage by allowing themselves to be configured in an optimal manner for a particular computation.

As these uncompression algorithms require some type of hardware acceleration (as do most graphics-intensive computations), we examined the design of optimal hardware for the task. We identified floating point multiplication to be the most computationally intensive aspect of uncompression---in particular, multiplication by a constant (for a

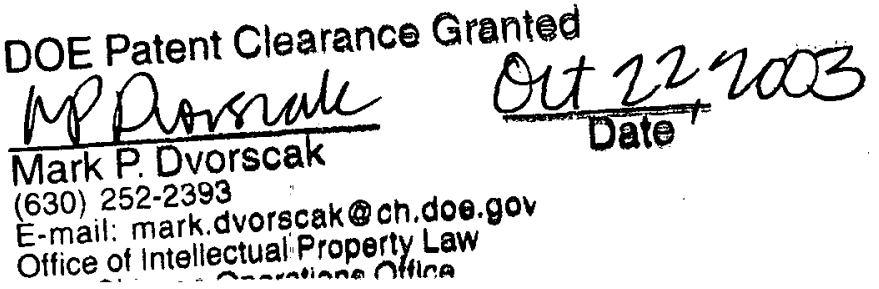




\section{DISCLAIMER}

This report was prepared as an account of work sponsored by an agency of the United States Government. Neither the United States Government nor any agency thereof, nor any of their employees, makes any warranty, express or implied, or assumes any legal liability or responsibility for the accuracy, completeness, or usefulness of any information, apparatus, product, or process disclosed, or represents that its use would not infringe privately owned rights. Reference herein to any specific commercial product, process, or service by trade name, trademark, manufacturer, or otherwise does not necessarily constitute or imply its endorsement, recommendation, or favoring by the United States Government or any agency thereof. The views and opinions of authors expressed herein do not necessarily state or reflect those of the United States Government or any agency thereof. 


\section{DISCLAIMER}

Portions of this document may be illegible in electronic image products. Images are produced from the best available original document. 
particular application). Given this, we have developed hardware designs for this computation, and mapped these designs onto configurable computing architectures. Experimentally, we have demonstrated that these new designs are faster than typical alternatives. In addition, we have recently developed theoretical results to predict and understand the performance of these algorithms on this novel architecture.

Extended SUMAA3d to support edge-based variables (as opposed to vertex-based variables). Support for these data structures allows the SUMAA3d programming environment to be used for numerical schemes based on finite-volume discretization methods (in addition to the finite-element based schemes already supported). In particular, this method provides support for work done at ANL on unstructured adaptive mesh methods for Raleigh-Taylor instabilities (see below).

The edge-based data structures were used in an implementation of a simulation of Raleigh-Taylor instabilities on unstructured meshes in SUMAA3d. This implementation used the edge-based data structures discussed above and is based on a piecewise constant finite-volume discretization method. The implementation is adaptive, and flux discontinuities are handled with a Reimann solver developed by Bruce Fryxell (Univ. of Chicago). References [1], [2], [6], [8]

Development of dynamic partitioning strategies: Explicit time-stepping methods are commonly used to solve transient, time-dependent problems. For problems involving small-scale features, adaptive unstructured mesh refinement can be effective.

Unfortunately, using such dynamic strategies on parallel computers can result in difficult load balancing issues because of the relatively high cost of the mesh partitioning to the time-step computation. We hàve developed a partitioning strategy that addresses this problem and demonstrates the effectiveness of this new approach, proposed method for two and three dimensional Raleigh-Taylor problems. This test problem is of particular interest to the DOE ASCI Center at the University of Chicago, and we have worked with members of this group on this application. Reference [12]

Mesh Optimization and untangling methods: An extension to our previous work on mesh optimization (with L. Frietag, ANL) is the development of mesh untangling algorithms for both sequential and parallel computers. Tangled meshes often arise in practice, for example, in the generation of meshes for complex computational domains or in the use of Lagragian (moving mesh) methods. The important aspect of this work is that it represents the first time the problem has been given any theoretical guarantees and an efficient, robust solution. The approach is based on the construction of local subproblems that are guaranteed to converge and the development of an efficient implementation for this subproblem solution (in this case, a specialized version of the simplex method).

Working with L. Freitag (Argonne), we have developed mesh untangling methods that are effective for quadrilateral meshes. These are local optization methods that can be used on both sequential and parallel computers. Tangled meshes arise in the generation of meshes for complex computational domains or in the use of Lagrangian (moving 
mesh) methods. This work is based on previous work on robust methods for untangling tetrahedral meshes. The approach is based on the construction of local subproblems that are guaranteed to converge and the development of an efficient implementation for this subproblem solution (in this case a specialized version of the simplex method). References [3], [4], [5], [7], [8], [10]

Scalable Particle Tracking Methods: Particle tracking methods are a versatile computational technique central to the simulation of a wide range of scientific applications. We have developed a mesh independent particle tracking software system based on "in-element" particle tracking. This approach is based on the assumption that particle trajectories are computed by problem data localized to individual elements. Adaptive mesh refinement is used to control the mesh discretization errors along computed characteristics of the particle trajectories. We have demonstrated the use of this approach in conjunction with SUMAA3d for advection dominated flow simulations. Reference [11]

Students supported:

Ruth Cheng, Third year graduate student, CSE Penn State

Chuang Li, Second year graduate student, CSE Penn State

Yanhua Yi, Second year PhD student, Virginia Tech

John Shifflet, undergraduate, Virginia Tech

Christopher Taylor, undergraduate, Virginia Tech

Zahi Nakad, Masters thesis student, Virginia Tech

Related Publications and Presentations:

1. "High Performance Applications on Reconfigurable Clusters", Zahi Nakad, Masters Theșis, ECE Department, Virginia Tech, 2000.

2. "Unstructured Mesh Computations on CCMs", M. Jones and K. Ramachandran, International Journal on Advances in Engineering Software, 2000.

3. "An Approach for Creating High-Quality Unstructured Meshes, Lori Freitag and Paul Plassmann, Invited Minisymposium Presentation, First SIAM Conference on Computational Science and Engineering, 2000.

4. "Optimization Approaches to Tetrahedral Mesh Improvement", Lori Freitag and Paul Plassmann, Invited Minisymposium Presentation, SIAM Annual Meeting, 2000. 
5. "A Parallel Mesh Improvement Algorithm", Mark Jones and Paul Plassmann, Invited Minisymposium Presentation, SIAM Annual Meeting, 2000.

6. "Mesh generation," Marshall Bern and Paul Plassmann, appeared in "Handbook of Computational Geometry," Jorg Sack and Jorge Urrutia, eds., Elsevier Science pp. 291-332 (2000).

7. "Local Optimization-based Simplicial Mesh Untangling and Improvement", Lori Freitag and Paul Plassmann, appeared in International Journal of Numerical Methods in Engineering, 49, pp. 109-125 (2000).

8. "Unstructured Mesh Computations on Networks of Workstations", Mark Jones and Paul Plassmann, appeared in Computer-Aided Civil and Infrastructure Engineering, 15 (3), pp. 196-208, (2000).

9. "A Parallel Algorithm for Mesh Smoothing", Lori Freitag, Mark Jones, and Paul Plassmann, appeared in SIAM Journal on Scientific Computing, 20, pp. 2023-2040 (1999).

10. "Local Optimization-based Untangling Algorithms for Quadrilateral Meshes", Lori Freitag and Paul Plassmann, Proceedings of the Tenth International Roundtable, Sandia National Laboratories, Albuquerque, NM, pp. 397-406, 2001.

11. "The Accuracy and Performance of Parallel In-Element Particle Tracking Methods", Jing-Ru Cheng and Paul Plassmann, Proceedings of the Tenth SIAM Conference on Parallel Processing for Scientific Computing, SIAM Publications, pp. 252-261, 2001.

12. "A Mesh Partitioning Strategy for Optimizing the Performance of Adaptive Explicit Time-Stepping Methods\}, Jing-Ru Cheng and Paul Plassmann, Proceedings of the Tenth SIAM Conference on Parallel Processing for Scientific Computing, SLAM Publications. pp. 527-536, 2001. 